\title{
Optimization of extraction parameters of phenolic antioxidants from defatted grape seeds flour by response surface methodology
}

\section{Sergey Gubsky, Maksym Labazov, Olga Samokhvalova, Nataliya Grevtseva, Olena Gorodyska}

\section{Kharkiv State University of Food Technology and Trade, Kharkiv, Ukraine}

Keywords:

Antioxidant

Polyphenols

Flavonoids

Grape seeds

Coulometry

Article history:

Received 23.02.2018

Received in revised form 04.06.2018

Accepted 27.12.2018

Corresponding

author:

Sergey Gubsky

E-mail:

s.gubsky@

hduht.edu.ua

DOI: $10.24263 / 2304-$

974X-2018-7-4-8

\section{Abstract}

Introduction. The optimization results of the conditions of liquid-solid extraction by wine industry waste water in the form of the defatted grape seeds flour in order to obtain the extract with high antioxidant capacity are conducted in this report.

Materials and methods. Total antioxidant capacity (TAC) and total phenolic content (TPC) of the samples were determined by the method of galvanostatic coulometric titration with electrogenerated bromine and spectrophotometric method using Folin-Ciocalteu reagent, respectively. TAC and TPC experimental values were presented in terms of the equivalent of gallic acid content (GAE) per unit mass of dry powder (DW).

Results and discussion. Response surface methodology (RSM) is used to search for optimal condition of solid-liquid extraction of phenolic compounds from defatted grape seeds flour under the influence of three factors: the temperature $\left(60-100^{\circ} \mathrm{C}\right)$, extraction time (90-150 $\mathrm{min}$ ) and liquid to solid ratio (60-100). The result showed that the phenolic substances yield in the set ranges is $1.20-2.64 \%$ with total antioxidant capacity of 17.71-36.78 mg GAE/g DW. Due to optimization procedure, it was determined that under optimal conditions (the temperature of $100{ }^{\circ} \mathrm{C}$; the extraction time is $131 \mathrm{~min}$; and the ratio of the extractive agent volume to the mass of the powder is 85 ) the maximum TAC of the extract of $37.04 \mathrm{mg}$ GAE/g DW is achieved. The maximum yield of phenolic substances of $2.646 \%$ was obtained under the following conditions: temperature of 100 ${ }^{\circ} \mathrm{C}$; extraction time is $117 \mathrm{~min}$; ; and the ratio of extraction agent volume to the mass of the powder is 93 . When optimization is used with two TAC and YPC response functions, the following optimal conditions were obtained: the temperature of $100{ }^{\circ} \mathrm{C}$, the extraction time is 123 minutes and the ratio of extractive agent volume to the mass of powder is 89 , at which $36.91 \mathrm{mg} \mathrm{GAE} / \mathrm{g}$ DW TAC and YPC values and $2.633 \%$ were obtained, respectively. The validation of obtained results showed their compliance within 3\% with experimental values.

Conclusion. The obtained results indicate the perspective of wine industry waste recycling in order to obtain the solid extract from defatted grape seeds flour as a source of biologically active substances of a phenolic nature with high antioxidant potential. 


\section{Introduction}

Plant objects are considered to be promising natural sources of antioxidants, and therefore, the number of studies on the development of plant-based additives technologies in the form of pastes, extracts and powders has recently increased. The supplementation of such additives allows obtaining functional nutrition products with high antioxidant potential as well as high biological and nutritional value.

Grape seeds are produced in large amounts as the wine industry waste products and are increasingly used to create food ingredients. This occurs due to the fact that it is a source of polyphenolic antioxidants - flavonoids, such as monomeric flavanols, dimeric, trimeric and polymeric procyanides and phenolic acids [1-3].

The extraction from raw plant materials is an important stage in phytochemical processing in order to optimize the concentration of biologically active compounds. The selection of a suitable solvent for the standardization of plant products is highly important in this process. Differences in the compounds structure determine their solubility in solvents of different polarity. Therefore, the type of extractive solvent can have substantial impact on the yield of the desired extracted compounds from the plant material. However, the selection of solvent is normally limited to water and ethanol or their mixture when using extracts for food purposes.

The selection of an appropriate withdrawal process and the optimization of various parameters are crucial for purposes of scaling and moving from the laboratory experiment to the industrial scale. According to the numerous data indicating that the optimal conditions for the extraction of phenolic compounds of some plant products are usually different for various plant matrices [4]. Extraction methods that are most commonly used include standard convection methods (maceration, percolation, infusion, decoction, hot continuous extraction) and non-convection methods (ultrasonically extraction (UEA), microwave radiation (MEA), pulse electrical discharge (PAED) and supercritical fluid extraction (SFE) [5]. Specified methods in different options of the experiment were used to conduct numerous studies on the extraction of polyphenols from grape waste for example, including grape seed [6-15].

The purpose of this study was the selection of optimal conditions for effective water solid-liquid extraction (SLE) of polyphenolic compounds from defatted grape seed flour in order to obtain an extract with the maximum antioxidant potential.

\section{Materials and methods}

\section{Chemicals}

The following chemicals used in this study are as follows: potassium bromide, sodium hydroxide, sodium carbonate (Reachim, Russia); sulphur acid (Sumychimprom, Ukraine); gallic acid (Sigma Aldrich, USA). All the chemicals used in this experiment were of analytical grade. The synthesis of Folin-Ciocalteu reagent was done according to the procedure [16]. All the chemicals used in this procedure were of analytical grade. For analysis $2 \mathrm{M}$ solution was used. For preparation of the solutions distilled water with electric conductivity no more 0.55 $\mathrm{mS} / \mathrm{m}$ was used. The conductivity was measured by a conductometer CEL-1M2 (Analitpribor, Georgia).

\section{Raw Material}

Defatted grape seeds flour (Oleo Vita trademark, Orion, Ukraine) was made from fresh grape seeds of unfermented squeezing in industrial conditions. A mixture of four grape varieties in equal proportions in mass, grown in the southern regions of Ukraine 
(Odessa region) was used as the raw material. Gently dried grape seeds at a temperature not more than $60{ }^{\circ} \mathrm{C}$ were subject to the thorough cleaning (separation).

Grape seed cake was obtained in the form of solid plates with its further fine grinding and crushing to fine powder (flour) in the process of cold pressing. The residual fat content in the flour was $8-9 \%$.

\section{Extraction Process}

Accurately weighted dried powder (according to ratio liquid to solid) were extracted with the solvent by $10 \mathrm{ml}$ in glass test tubes (total volume $15 \mathrm{ml}$ ) with screw caps. Distilled water was used as the extractive agent. All the time the tubes were shaken. The extraction was performed in the dark and at corresponding temperature, a solid-liquid ratio and the extraction time. The tubes were placed in a thermostat at a set temperature for the extraction time. The liquid after extraction was separated from solids by centrifugation at $6.0 \mathrm{~g}$ for $10 \mathrm{~min}$ (OPn8UHL4.2, Russia).

The solutions were kept at a constant temperature using the thermostat $1 \mathrm{TZH}-0.03$ (Russia). The temperature in this device was maintained at an accuracy of $0.2{ }^{\circ} \mathrm{C}$ and determined by the sensor SM60-Pt1000 (Yokogawa Europa, Holland) with a precision of 0.1 ${ }^{\circ} \mathrm{C}$.

The samples were weighed on laboratory scales balance CBA-300-0.005 (T-Scale, China) with accuracy of $5 \mathrm{mg}$ and on analytical laboratory scales balance VLR-200 (Gosmetr, Russia) with accuracy up to $0.1 \mathrm{mg}$.

\section{Determination of the Total Antioxidant Capacity}

TAC of simples was determined by the reaction with electronegative bromine [17]. In this research using the same method that was detailed $[18,19]$. The experimental data of coulometric titration were used to calculate the TAC, as the electricity quantity Q, spent for titration per $100 \mathrm{~g}$ of the sample and it were calculated by expression:

$$
T A C=\frac{100 \mathrm{Itm}_{\text {solution }}}{m_{\text {al }} m_{\text {sample }}},
$$

where $m_{\text {sample }}$ is the weight of the sample (dry powder of plant or candy caramel), $m_{\text {solution }}$ is the total weight of the solution for candy caramel or of the extract for powder of plant. Values of TAC in gallic acid equivalent (mg GAE/100 g sample) were calculated. For aqueous solutions of gallic acids the coefficients of the linear regression were determined in [18].

\section{Determination of the Total Phenolic Content}

The concentration of phenolic compounds in samples was estimated using a modified spectrophotometric Folin-Ciocalteu method according Singleton and Rossi [16] with the transition from volume to weight of the aliquot portion. Briefly, $0.1 \mathrm{~g}$ of extract, standard or blank solution was mixed with $0.5 \mathrm{~g}$ of Folin-Ciocalteu's reagent and $2.0 \mathrm{~g}$ water. A sample of extracts was previously diluted in 10 times. After $8 \mathrm{~min}, 1.5 \mathrm{~g}$ of sodium carbonate $20 \%$ $(\mathrm{w} / \mathrm{w})$ solution was added to the mixture and adjusted to $10.0 \mathrm{~g}$ with distilled water. Mixture was incubated for $30 \mathrm{~min}$ in thermostat at $45{ }^{\circ} \mathrm{C}$ temperature. Finally, measurement of absorbance was carried out in spectrophotometer SF-46 (Lomo, Russia) and modernized and connected with a computer Specord UV-VIS (JenaAnalytik, Germany) at wavelength of 765 $\mathrm{nm}$ against a blank sample.

Gallic acid was used as a standard. The TPC values were expressed as mg of gallic acid equivalents (GAEs) per g of dry weight (DW) of plant. A $1000 \mathrm{mg} / \mathrm{kg}$ stock solution of gallic 
acid was prepared by dissolving $0.1 \mathrm{~g}$ of gallic acid in $100 \mathrm{~g}$ of distilled water. Working standard solutions of gallic acid at five different concentration levels $(25,50,100,250$ and $500 \mathrm{mg} / \mathrm{kg}$ ) were prepared by dilution of the stock solution. The gallic acid calibration curve was constructed in the range of $25-500 \mathrm{mg} / \mathrm{kg}$ and used to calculate linear regression models [18].

The yield (\%) of the extraction of phenolic compound (gram to gram dry weight of powder) was defined as :

$$
Y P C=\frac{T P C}{1000} 100 \%,
$$

\section{Experimental Design}

A response surface methodology (RSM) as a tool for optimization in this research was applied [20]. A tree level, three variable Box-Behnken design [21] was applied to determine the best combinations TAC and TPC (maximum value) of extraction variables for the extraction from defatted grape seeds flour.

Table 1

Independent variables and their levels employed in a Box-Behnken design for optimization of defatted grape seed flour extracts

\begin{tabular}{|c|c|c|c|c|}
\hline \multirow{3}{*}{ Independent variables } & \multirow{3}{*}{ Symbol } & \multicolumn{3}{|c|}{ Coded levels } \\
\hline & & -1 & $\mathbf{0}$ & 1 \\
\hline & & \multicolumn{3}{|c|}{ Natural levels } \\
\hline Temperature, ${ }^{\circ} \mathrm{C}$ & Temp & 60 & 80 & 100 \\
\hline Time, $\min$ & Time & 90 & 120 & 150 \\
\hline $\begin{array}{l}\text { Ratio volume of liquid to } \\
\text { weigth of solid powder }(\mathrm{v} / \mathrm{w}), \mathrm{ml} / \mathrm{g}\end{array}$ & $\mathrm{R} \mathrm{L} / \mathrm{S}$ & 60 & 80 & 100 \\
\hline
\end{tabular}

Three independent variables selected for this study were the extraction temperature (T), the extraction time $(\mathrm{t})$, and ratio volume of liquid to weight of solid powder (R L/S) (Table 1).

Preliminary trials showed as the values of responses changed with increasing temperature, time and ratio $\mathrm{L} / \mathrm{S}$; therefore, the optimal levels were selected as center points in the designed experiment. The factorial design consisted of requires an experiment number according to $\mathrm{N}=2 \mathrm{k}(\mathrm{k}-1)+\mathrm{cp}$, where $\mathrm{k}$ is the number of factors $(\mathrm{k}=3)$ and $(\mathrm{cp})$ is the number of the central points eight factorial points, and three center points leading to 15 sets of experiments [20].

Regression analysis was performed on the data of dependent variables as effected by the extraction conditions and was fitted into an empiric second order polynomial model as shown in the following equation:

$$
Y=\beta_{0}+\sum \beta_{i} X_{i}+\sum \beta_{i i} X_{i}^{2}+\sum \beta_{i j} X_{i} X_{j},
$$

where $\mathrm{Y}$ is the predicted response; $\beta_{0}, \beta_{i}, \beta_{i i}, \beta_{i j}$ are the interception coefficient, the linear terms, the quadratic terms and the interaction terms, respectively; $X_{i}, X_{j}$ are the actual levels of the independent variables.

\section{Statistical analysis}

Determination of the experimental values were done for a number of parallel measurements $(n=4)$. The Student's t-test permitted us to check the statistical significance of the regression coefficients. A $p<0.05$ was considered as statistically significant. The Fisher's test for analysis of variance (ANOVA) was performed on experimental data to evaluate the 
statistical significance of the model. The the Design Expert software trial version 11.0 (StatEase, USA) were employed for the regression analysis and the graphical optimization, respectively.

The models of each response for full-factorial design were expressed in terms of actual variables and without taking into account the statistically insignificant terms.

\section{Results and discussion}

\section{Fitting the model of the extraction process from defatted grape seed flour}

The experimental data of dependence of TAC and YPC water extract of grape seeds flour from the investigated factors are shown in Table 2.

These experimental data were processed in the framework of analysis of variance (ANOVA) using the model of the average, linear, mixed and quadratic models. As it is commonly known that, ANOVA is a statistical analysis method for assessing the significance of experimental data and for adequacy analysis of the fitting model [20]. Analysis of obtained calculations showed that the most adequate description of TAC and YPC experimental values is achieved in a quadratic model. ANOVA data results for a quadratic model describing the dependence of total antioxidant capacity from the investigated factors are given in Table 3 .

Table 2

Experimental of the three-level and three-variable Box-Behnken design and TAC, TPC and yield of polyphenolic compound of defatted grape seed flour extracts

\begin{tabular}{|c|c|c|c|c|c|c|c|c|}
\hline \multirow[b]{2}{*}{ Run } & \multicolumn{3}{|c|}{ Code pattern } & \multirow{2}{*}{$\begin{array}{c}\text { Temp, } \\
{ }^{\circ} \mathrm{C} \\
\text { (A) }\end{array}$} & \multirow{2}{*}{$\begin{array}{l}\text { Time, min } \\
\text { (B) }\end{array}$} & \multirow{2}{*}{$\begin{array}{c}R \text { L/S } \\
\text { (C) }\end{array}$} & \multicolumn{2}{|c|}{ Experimental values } \\
\hline & Temp & Time & $\mathbf{R} \mathbf{L} / \mathbf{S}$ & & & & $\begin{array}{c}\text { YPC, } \\
\%\end{array}$ & $\begin{array}{c}\text { TAC, } \\
\text { GAE mg/ } \\
\text { g DW }\end{array}$ \\
\hline 1 & 0 & 1 & -1 & 80 & 150 & 60 & 1.625 & 22.93 \\
\hline 2 & -1 & 1 & 0 & 60 & 150 & 80 & 1.492 & 19.62 \\
\hline 3 & 0 & -1 & -1 & 80 & 90 & 60 & 1.522 & 21.89 \\
\hline 4 & -1 & 0 & -1 & 60 & 120 & 60 & 1.394 & 17.71 \\
\hline 5 & 1 & 0 & 1 & 100 & 120 & 100 & 2.550 & 35.51 \\
\hline 6 & 1 & 1 & 0 & 100 & 150 & 80 & 2.526 & 36.78 \\
\hline 7 & 0 & 0 & 0 & 80 & 120 & 80 & 1.882 & 25.98 \\
\hline 8 & -1 & 0 & 1 & 60 & 120 & 100 & 1.428 & 20.19 \\
\hline 9 & 0 & -1 & 1 & 80 & 90 & 100 & 1.757 & 24.95 \\
\hline 10 & 0 & 0 & 0 & 80 & 120 & 80 & 1.893 & 26.02 \\
\hline 11 & 0 & 0 & 0 & 80 & 120 & 80 & 1.914 & 26.02 \\
\hline 12 & -1 & -1 & 0 & 60 & 90 & 80 & 1.144 & 18.14 \\
\hline 13 & 1 & -1 & 0 & 100 & 90 & 80 & 2.600 & 35.72 \\
\hline 14 & 1 & 0 & -1 & 100 & 120 & 60 & 2.288 & 35.73 \\
\hline 15 & 0 & 1 & 1 & 80 & 150 & 100 & 1.940 & 27.20 \\
\hline
\end{tabular}

The coefficients $R^{2}$ and $R_{\text {adjusted }}^{2}$ are equal to 0.9944 и 0.9843 , respectively, according to the results from Table 3 . These values indicate that the model adequately describes the experimental data obtained and that they are well coordinated with their predicted values. The model is statistically significant because the p value is less than 0.0001 . The obtained value of the variation coefficient (C.V. $=3.19 \%)$ is quite low and indicates the reliability of the experimental data. 
Table 3

Analysis of variance (ANOVA) of the fitted quadratic polynomial model for the TAC of defatted grape seed flour extracts

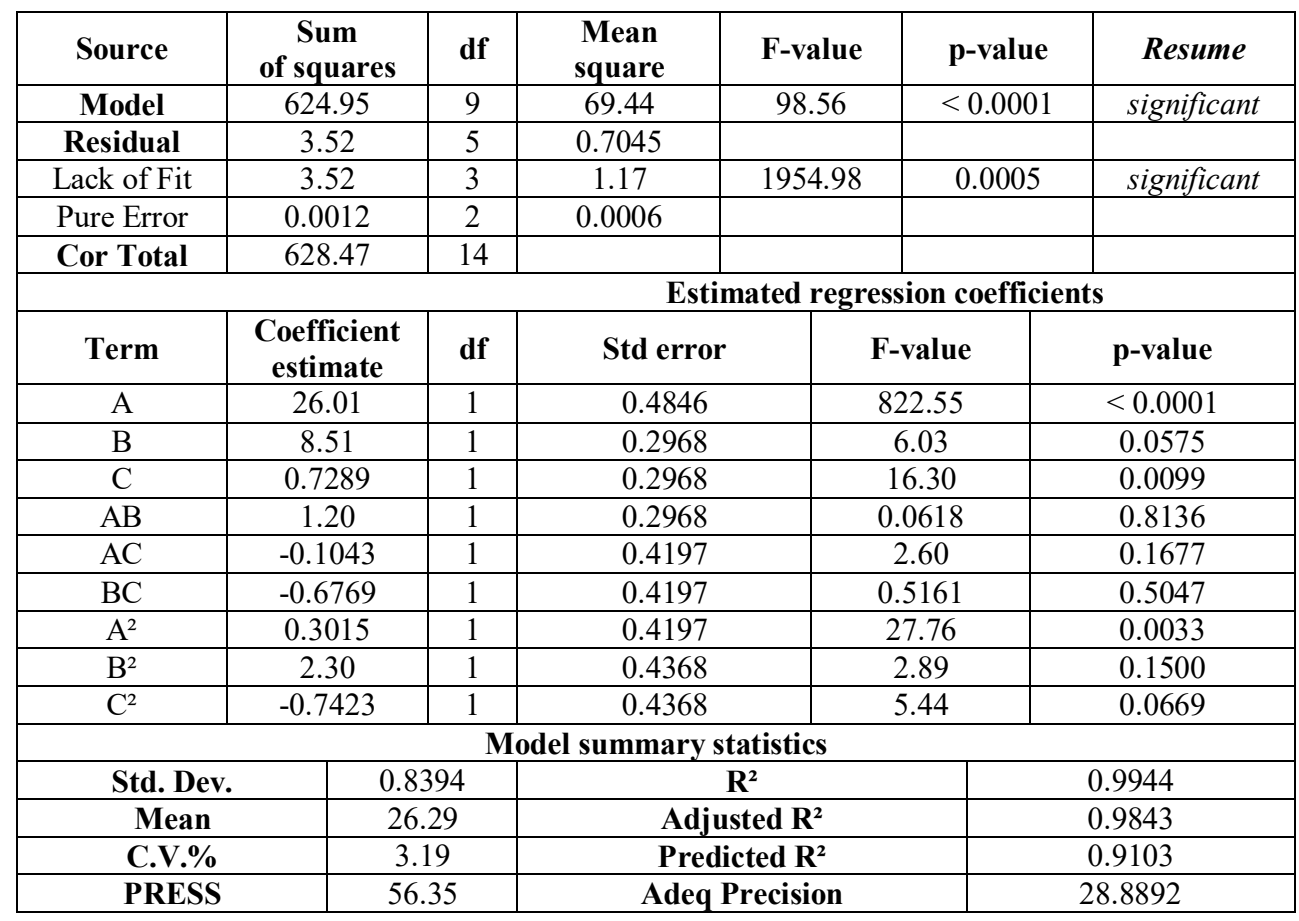

The Fisher criterion value of $\mathrm{F}$ at 98.56 level implies that the model is statistically significant. The obtained values of $\mathrm{p}$ criterion that are less than 0.0500 indicate the significance of the calculated coefficients $\mathrm{A}, \mathrm{C}, \mathrm{A}^{2}$ of equation (3). The calculated $\mathrm{F}$ values for Lack of Fit are also statistically significant. The predicted value of Predicted $R^{2}=0.9103$ is in necessary compliance with Adjusted $\mathrm{R}^{2}=0.9843$, since their difference is less than 0.2 . The value of AdeqPrecision, expressing the signal-to-noise ratio, and equal to 28.8892 is greater than the indicative value 4 , which indicates data adequacy.

Similar conclusions can be made with respect to the model describing the phenolic compounds output in the extraction process (Table 4). The adequacy of the experimental data description on the phenolic substances output is achieved by indicated model, as evidenced by the high correlation coefficients $R^{2}$ and $R_{\text {adjusted }}^{2}$ that are equal to 0.9874 и 0.9646 , respectively, and the $\mathrm{p}$ value that is equal to 0.0003 . The reliability of the experimental data was also confirmed by the value of factor at the level of C.V. $=4.59 \%$.

The adequacy of the experimental data description on the phenolic substances output is achieved by indicated model, as evidenced by the high correlation coefficients $R^{2}$ and $R_{\text {adjusted }}^{2}$ that are equal to 0.9874 и 0.9646 , respectively, and the $\mathrm{p}$ value that is equal to 0.0003 . The reliability of the experimental data was also confirmed by the value of factor at the level of C.V. $=4.59 \%$.

The value of Fisher's criterion $\mathrm{F}$ at the level of 43.37 is lower than in the previous review, and it demonstrates better statistical significance. The obtained values of $\mathrm{p}$ criterion are less 
than 0.0500 and they indicate the significance of the calculated coefficients $A, C, A^{2}$ of equation (3).

The calculated $\mathrm{F}$ values for Lack of Fit are also statistically significant. The predicted value of Predicted $R^{2} 0.8001$ is in reasonable compliance with Adjusted $R^{2} 0.9646$. The value of Adeq Precision, which represents a signal-to-noise ratio, is 20.6011 greater than the indicative value 4 , which indicates data adequacy.

Table 4

Analysis of variance (ANOVA) of the fitted quadratic polynomial model for the YPC of of defatted grape seed flour extracts

\begin{tabular}{|c|c|c|c|c|c|c|}
\hline Source & $\begin{array}{c}\text { Sum } \\
\text { of squares }\end{array}$ & df & $\begin{array}{c}\text { Mean } \\
\text { square }\end{array}$ & F-value & p-value & Resume \\
\hline Model & 2.86 & 9 & 0.3175 & 43,37 & 0,0003 & significant \\
\hline Residual & 0.0366 & 5 & 0.0073 & & & \\
\hline Lack of Fit & 0.0361 & 3 & 0.0120 & 45,55 & 0,0216 & significant \\
\hline Pure Error & 0.0005 & 2 & 0.0003 & & & \\
\hline Cor Total & 2.89 & 14 & & & & \\
\hline \multicolumn{7}{|c|}{ Estimated regression coefficients } \\
\hline Term & $\begin{array}{c}\text { Coefficient } \\
\text { estimate }\end{array}$ & df & Std error & F-value & p-value \\
\hline A & 1.90 & 1 & 0.0494 & 346,84 & $<0.0001$ \\
\hline B & 0.5634 & 1 & 0.0303 & 5,35 & 0,0687 \\
\hline C & 0.0700 & 1 & 0.0303 & 12,21 & 0,0174 \\
\hline AB & 0.1057 & 1 & 0.0303 & 6,10 & 0,0566 \\
\hline AC & -0.1056 & 1 & 0.0428 & 1,76 & 0,2417 \\
\hline BC & 0.0568 & 1 & 0.0428 & 0,2210 & 0,6581 \\
\hline$A^{2}$ & 0.0201 & 1 & 0.0428 & 7,76 & 0,0386 \\
\hline$B^{2}$ & 0.1241 & 1 & 0.0445 & 3,22 & 0,1325 \\
\hline$C^{2}$ & -0.0800 & 1 & 0.0445 & 5,62 & 0,0639 \\
\hline \multicolumn{7}{|c|}{ Model summary statistics } \\
\hline Std. Dev. \\
\hline Mean
\end{tabular}

The Pearson's correlation coefficient for actual and predicted TAC and YPC values at a level of 0.9972 and 0.9936 , respectively, indicates the possibility of using the models obtained to predict data on antioxidant capacity and polyphenolic compounds output for defatted grape seed flour when conducting solid-liquid extraction with water.

The following forecast models for describing the properties studied at the code level, which are described by expressions (4) and (5), are subject to proceed to, summarizing all the above:

$$
\begin{array}{r}
\text { TAC }(\mathrm{mg} / \mathrm{g} \mathrm{DW})=26.01+8.51 \mathrm{~A}+0.7289 \mathrm{~B}+1.20 \mathrm{C}-0.1043 \mathrm{AB}- \\
-0.6769 \mathrm{AC}+0.3015 \mathrm{BC}+2.30 \mathrm{~A}^{2}-0.7423 \mathrm{~B}^{2}-1.02 \mathrm{C}^{2} \\
\text { YPC }(\mathrm{mg} / \mathrm{g} \mathrm{DW})=1.90+0.5634 \mathrm{~A}+0.0700 \mathrm{~B}+0.1057 \mathrm{C}-0.1056 \mathrm{AB}+ \\
+0.0568 \mathrm{AC}+0.0201 \mathrm{BC}+0.1241 \mathrm{~A}^{2}-0.0800 \mathrm{~B}^{2}-0.1056 \mathrm{C}^{2}
\end{array}
$$




\section{Effect of process variables on Total Antioxidant Capasity}

The effect of independent variables of temperature, extraction time and ratio on the total antioxidant activity is shown in Figure 2. As seen from the scan, TAC value under the effect of independent variables varies within 17.71-36.78 GAE mg/g DW.

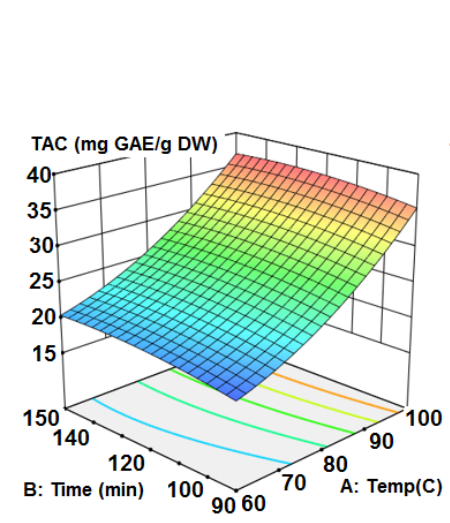

a

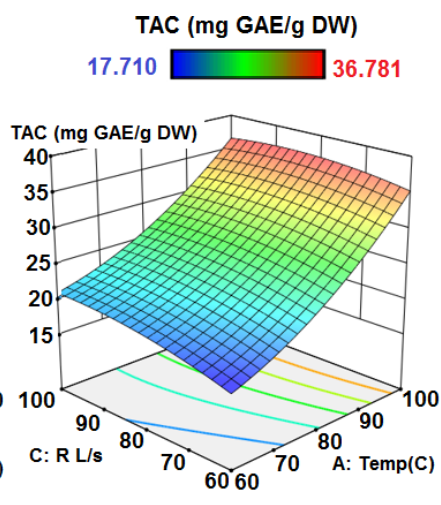

b

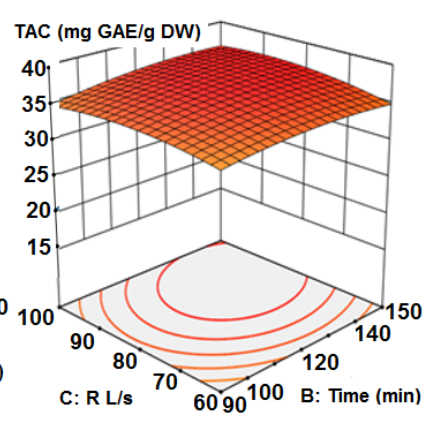

c

Figure 2. The 3D and 2D response surfaces profiles of TAC as affected by independent variables at optimal conditions:

ratio $\mathrm{S} / \mathrm{L} 85$ (a), time $131 \mathrm{~min}(\mathrm{~b})$ and temperature $100^{\circ} \mathrm{C}$ (c)

The analysis of three-dimensional surfaces of the basic property dependence on a pair of variables at a fixed optimal value of the third parameter has allowed establishing the following consistent pattern:

- $\quad$ an increase in temperature from 60 to $100^{\circ} \mathrm{C}$ leads to steady increase in TAC value regardless of the nature of the second independent parameter and the fixed value of the third parameter (Figure 2, a, b) and the achievement of the maximum TAC $=36.78 \mathrm{GAE}$ $\mathrm{mg} / \mathrm{g} \mathrm{DW}$ at a temperature of $100{ }^{\circ} \mathrm{C}$

- when fixing the temperature and considering the dependence of TAC value on extraction time and $\mathrm{R} \mathrm{L} / \mathrm{S}$, a surface with strongly pronounced single point of extremum, which corresponds to the maximum TAC value at a fixed temperature of $100{ }^{\circ} \mathrm{C}$ (Figure $2, \mathrm{c})$ is obtained.

\section{Effect of process variables on Total Phenolics Content}

The effect of temperature independent variables, extraction time and ratio $\mathrm{L} / \mathrm{S}$ on the phenolic compounds yield is shown in Figure 3. As can be seen from the scan, the output value of phenolic compound varies under the effect of independent variables within $1.14-2.60 \%$. The effect of temperature, as in the case of the total antioxidant capacity, leads to a monotonic increase in the YPC value on the entire studied variation interval from 60 to $100{ }^{\circ} \mathrm{C}$, regardless of the variation of the second parameter (Figure 3, a, b). When the temperature is fixed at the optimal level and the other two parameters variation, the pronounced extreme dependence on the extraction time on all curves, regardless of the value of R L/S (Figure 3, c) is obtained. 
The monotonous growth of YPC with an increase in the extraction time without reaching an extremum is observed on projection curves up to $\mathrm{R} L / \mathrm{S} 90$ values. The pronounced extreme dependence is weakly detected only after the value of 90 .

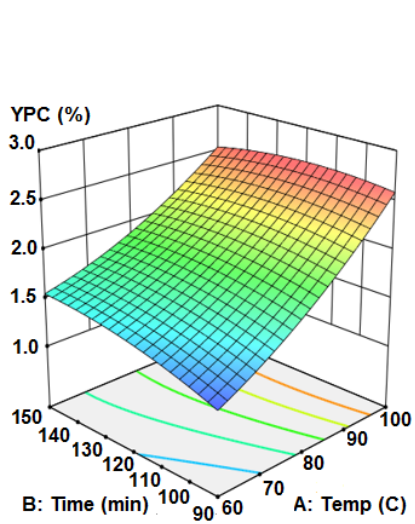

a

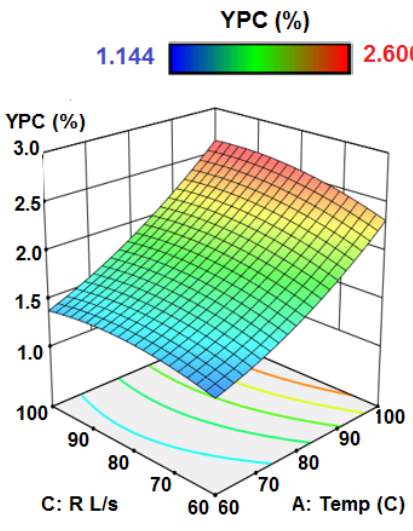

b

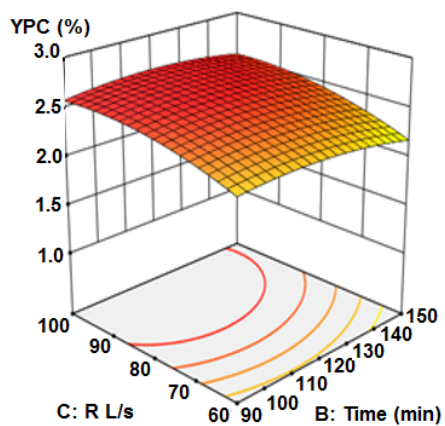

c

Figure 3. The 3D and 2D response surfaces profiles of YPC as affected by independent variables at optimal conditions:

ratio $\mathrm{S} / \mathrm{L} 93$ (a), time $117 \mathrm{~min}(\mathrm{~b})$ and temperature $100{ }^{\circ} \mathrm{C}$ (c)

\section{Optimum conditions for SLE from defatted grape seeds flour by water}

The regression models obtained from Eqs. 4 and 5 were used to determine optimum values of extraction temperature, time and ratio liquid to solid by using optimization procedures. The objective function (Q) in this optimization was defined to maximize simultaneously the TAC as well as to maximize the yield of phenolic compounds extract with subject to temperature range $(\mathrm{A})$, time $(\mathrm{B})$, and ratio solid to liquid $(\mathrm{C})$ :

$$
\max Q=\left\{\begin{array}{c}
\text { TAC } \\
\text { YPC } \\
\text { TAC }+ \text { YPC }
\end{array}, \quad T A C, Y P C=f(A, B, C), \quad-1<A, B, C<1 .\right.
$$

The optimal conditions are fairly simply and efficiently determined using the RSM methodology mentioned above for a single-response function [20]. TAC and YPC 37.04 and 2.646 values were obtained as a result of carrying out the optimization procedure, respectively (Table 4).

It should be noted that the completed optimization procedure using models (equations 4 and 5) makes it possible to obtain more than ten sets of optimal parameters at which the values of the optimal function are in the $1 \%$ interval of variation from the maximum value. Although, the differences in the optimal values of the parameters $\mathrm{A}, \mathrm{B}$ and $\mathrm{C}$ are also practically in the same variation interval.

Estimated optimum conditions of responses TAC and YPC

Table 4

\begin{tabular}{|c|c|c|c|c|c|c|}
\hline & $\mathbf{T}^{\circ}{ }^{\circ} \mathbf{C}$ & $\mathbf{t}, \mathbf{m i n}$ & $\mathbf{R ~ L} / \mathbf{S}$ & TAC, $\mathbf{~ m g ~ G A E} / \mathbf{g}$ DW & YPC, $\%$ & Desirability \\
\hline $\mathrm{TAC}$ & 100 & 131 & 85 & 37.04 & & 0.9585 \\
\hline $\mathrm{YPC}$ & 100 & 117 & 93 & & 2.646 & 0.9537 \\
\hline TAC + YPC & 100 & 123 & 89 & 36.91 & 2.633 & 0.9504 \\
\hline
\end{tabular}


It should be noted that the completed optimization procedure using models (equations 4 and 5) makes it possible to obtain more than ten sets of optimal parameters at which the values of the optimal function are in the $1 \%$ interval of variation from the maximum value. Although, the differences in the optimal values of the parameters A, B and C are also practically in the same variation interval.

Under multivariate consideration, the Derringer function or the desirability function (Desirability) were used, preferring a set with larger value (Figure 4) as a final selection criterion of the optimal conditions set.

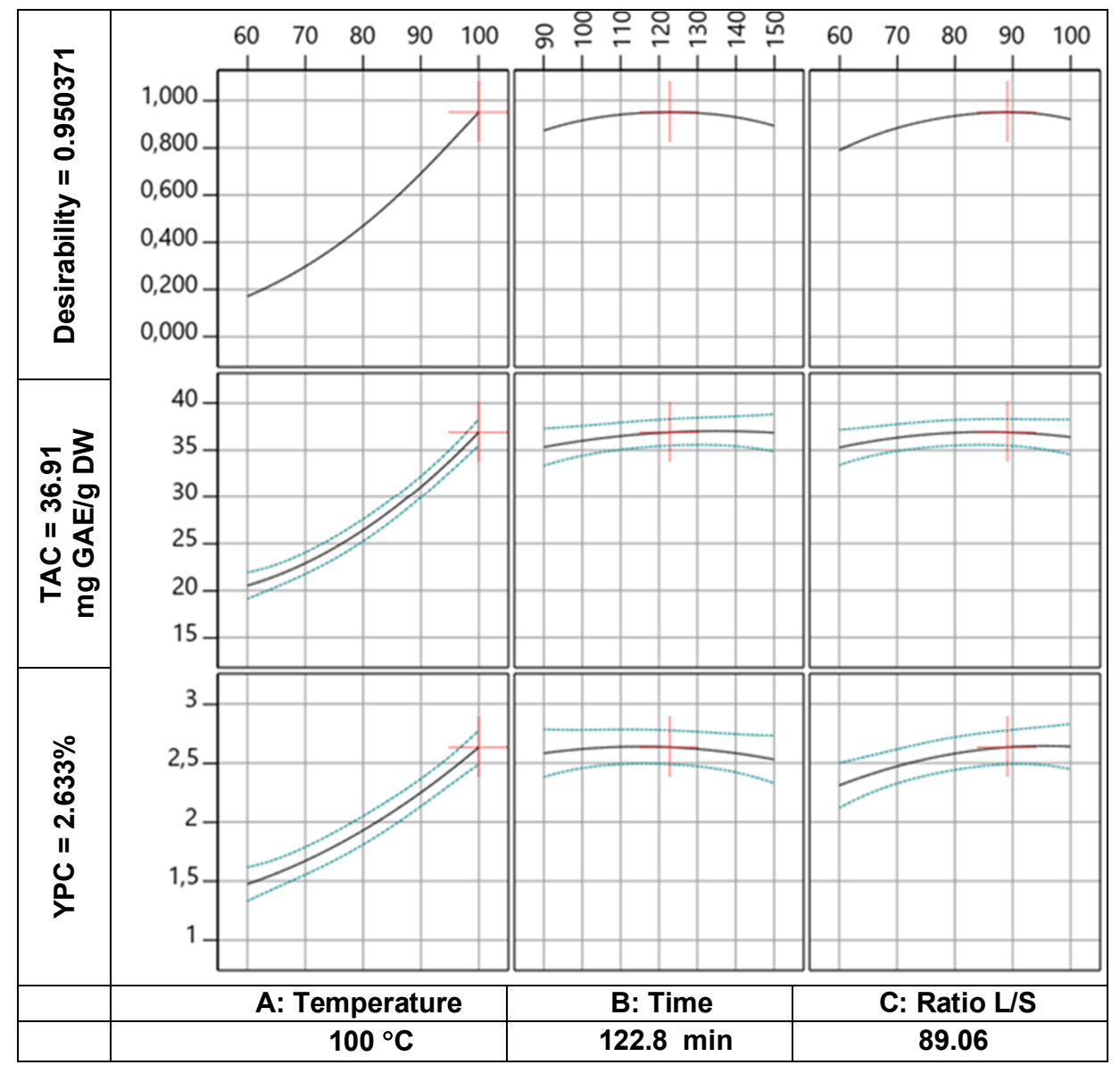

Figure 4. Overall optimum conditions of solid-liquid extraction of phenolic antioxidants from defatted grape seed flour

This function was used as a selection criterion of compromise solution in the multicriteria optimization technology, in our case with two TAC and YPC response functions. It should be noted that the desirability function is the most important and most frequently used in optimization procedures with multiple response functions [20]. The corresponding optimization algorithm of extraction procedure conditions is effective and economical, when solving the tasks of the output maximization of necessary components from the plant matrix based on several response functions [22]. 
The results of optimization process of the combination of TAC and YPC values within the variation range of independent extraction variables are presented in Figure 4. The maximum value of the desirability function $(0.9504)$ is reached at a temperature of $100{ }^{\circ} \mathrm{C}$, extraction time of 123 minutes and the ratio of the volume of water to the mass of powder 89 (Table 4).The obtained values of antioxidant capacity and total content of phenolic compounds yield is $36.91 \mathrm{mg} \mathrm{GAE} / \mathrm{g}$ DW and $2.633 \%$ less than the similar for each of the properties optimal conditions are only at the level of $0.5 \%$.

Table 5

Comparison between predicted and experimental values in optimal conditions of extractive process (temperature $100{ }^{\circ} \mathrm{C}$, time $123 \mathrm{~min}$, ratio $\mathrm{L} / \mathrm{S} \mathrm{89}$ )

\begin{tabular}{|l|c|c|c|}
\hline \multicolumn{1}{|c|}{ Value } & Predicted & Experimental & $\mathbf{\delta , \%}$ \\
\hline TAC, mg GAE/g DW & 36.91 & 36.45 & 1.2 \\
YPC,\% $\%$ & 2.633 & 2.705 & 2.7 \\
\hline
\end{tabular}

\section{Model validation}

The obtained models of TAC and TPC were verified by comparing the predicted data with experimental data. Table 5 shows the comparison between the predicted and experimental values for each response studied at conditions: temperature $-100^{\circ} \mathrm{C}$, time 123 $\min$ and ratio $\mathrm{L} / \mathrm{S} 89$.

As show Table 5, Experimentally, the values in all three cases are in good agreement with theoretical values within $3 \%$. This can be considered a satisfactory result.

\section{Correlation TAC and TPC values}

The various mechanisms of oxidation-reduction reactions, simulating the effect of radical oxidation are used in methods for studying antioxidant properties [23, 24]. The method of galvanostatic coulometry with electrogenerated bromine was used as a method for evaluating antioxidant capacity in this study. The procedure is based on the interaction of antioxidants with bromine and allows evaluating the integral antioxidant capacity of a wide range of materials, including plant materials and materials of a biological nature [25]. The methodology for determining the total content of polyphenols is widely used to estimate the total content of polyphenolic antioxidants, unlike the previous one [24]. Although the different mechanism of reactions effect on which these methodologies are based, there is a quite high positive correlation between the obtained data (Figure 5).

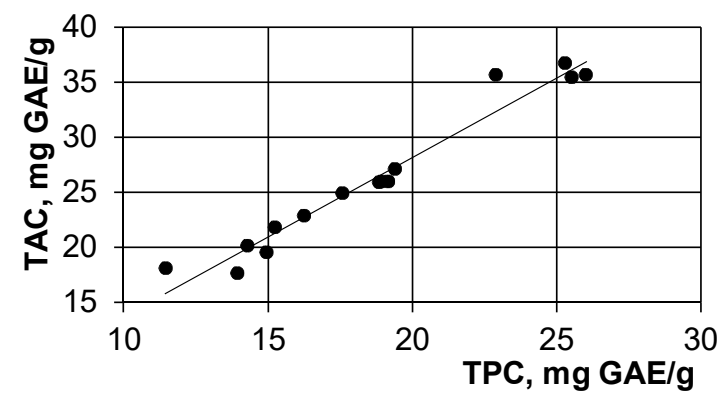

Figure 5. Correlation plots for TAC vs. TPC of water extracts from defatted grape seed flour, $\mathbf{R}^{2}=\mathbf{0 . 9 5 8 7}$ 
Similar regularities were obtained for confectionery products with plant extracts [18]. Data concerning the correlation of TAC and TPC values is another proof that the coulometric titration methodology with electrogenerated bromine is sufficiently adequate for the purposes of determining the total antioxidant capacity in extracts and food samples based on them, with the prevailing content of phenolic compounds as antioxidants.

\section{Conclusion}

The following conclusions can be drawn, based on the results obtained:

1. As a result of optimization procedure, it has been determined, that upon optimal conditions, in particular the temperature of $100{ }^{\circ} \mathrm{C}$, the extraction time of 131 minutes and the ratio of the extractive agent volume to the mass of powder of 85, the maximum total antioxidant capacity of the extract, that is equal to $37.04 \mathrm{mg} \mathrm{GAE} / \mathrm{g}$ DW can be obtained. The maximum output of phenolic substances of $2.646 \%$ was obtained under the following conditions: the temperature of $100{ }^{\circ} \mathrm{C}$, the extraction time of 117 minutes and the ratio of the extractive agent volume to the mass of powder of 93 .

2. The usage of Derringer function as a selection criterion of compromise solution in a multicriteria optimization technology with two TAC and YPC response functions, makes it possible to quickly and effectively predict the optimal conditions of the extraction procedure: the temperature of $100^{\circ} \mathrm{C}$, the extraction time of 123 minutes and the ratio of the extractive agent volume to the mass of powder of 89 - the conditions under which the values of TAC and YPC 36,91 $\mathrm{mg} \mathrm{GAE} / \mathrm{g}$ DW and 2,633\%, were obtained. The validation of obtained results showed their compliance within $3 \%$ with experimental values.

3. The high correlation value between the total antioxidant capacity and the total content of phenolic substances indicates that the galvanostatic coulometric titration with electrogenerated bromine is an appropriate procedure for the purpose of quantitative evaluation of total antioxidant potential of the phenolic compounds in plant extracts.

\section{References}

1. Ma Z., \& Zhang H. (2017), Phytochemical Constituents, Health Benefits, and Industrial Applications of Grape Seeds: A Mini-Review, Antioxidants, 6(3), pp. 71.

doi:10.3390/antiox6030071

2. Li L., \& Sun B. (2017), Grape and wine polymeric polyphenols: Their importance in enology, Critical Reviews in Food Science and Nutrition, pp. 1-17. doi:10.1080/10408398.2017.1381071

3. Guendez R., Kallithraka S., Makris D. P., \& Kefalas P. (2005), Determination of low molecular weight polyphenolic constituents in grape (Vitis vinifera sp.) seed extracts: Correlation with antiradical activity, Food Chemistry, 89(1), pp. 1-9. doi:10.1016/j.foodchem.2004.02.010

4. Watson R. R. (2014), Polyphenols in Plants: Isolation, Purification and Extract Preparation, Elsevier. doi:10.1016/C2011-0-08711-2

5. Lebovka F., Vorobiev N., \& Chemat E. (2011), Enhancing Extraction Processes in the Food Industry (Vol. 20114942), CRC Press. doi:10.1201/b11241

6. Casazza A. A., Aliakbarian B., De Faveri D., Fiori L., \& Perego P. (2012), Antioxidants from winemaking wastes: A study on extraction parameters using response surface methodology, Journal of Food Biochemistry, 36(1), pp. 28-37. doi:10.1111/j.1745-4514.2010.00511.x

7. Casazza A. A., Aliakbarian B., Mantegna S., Cravotto G., \& Perego P. (2010), Extraction of phenolics from Vitis vinifera wastes using non-conventional techniques, Journal of Food Engineering, 100(1), pp. 50-55. doi:10.1016/j.jfoodeng.2010.03.026

8. Ma-Hock L., Gamer A. O., Landsiedel R., Leibold E., Frechen T., Sens B., ... Van Ravenzwaay 
B. (2007), Generation and characterization of test atmospheres with nanomaterials, Inhalation Toxicology, 19(10), pp. 833-848. doi:10.1080/08958370701479190

9. Casazza A. A., Aliakbarian B., \& Mura M. (2015), Polyphenols from Grape and Apple Skin : a Study on Non- Conventional Extractions and Biological Activity on Endothelial Cell Cultures, 44(Young 1995), pp. 205-210. doi:10.3303/CET1544035

10. Tarley C. R. T., Silveira G., dos Santos W. N. L., Matos G. D., da Silva E. G. P., Bezerra M. A., Ferreira S. L. C. (2009), Chemometric tools in electroanalytical chemistry: Methods for optimization based on factorial design and response surface methodology, Microchemical Journal, 92(1), pp. 58-67. doi:10.1016/j.microc.2009.02.002

11. Garcia-Salas P., Morales-Soto A., Segura-Carretero A., \& Fernández-Gutiérrez A. (2010), Phenolic-compound-extraction systems for fruit and vegetable samples, Molecules, 15(12), pp. 8813-8826. doi:10.3390/molecules 15128813

12. Vorobiev E., \& Lebovka N. (2016), Application of Pulsed Electric Energy for Grape Waste Biorefinery (D. Miklavcic, Ed.), Handbook of Electroporation, Cham: Springer International Publishing. doi:10.1007/978-3-319-26779-1

13. Ghafoor K., Choi Y. H., Jeon J. Y., \& Jo I. H. (2009), Optimization of ultrasound-assisted extraction of phenolic compounds, antioxidants, and anthocyanins from grape (Vitis vinifera) seeds, Journal of Agricultural and Food Chemistry, 57(11), pp. 4988-4994. doi:10.1021/jf9001439

14. Ashraf-Khorassani M., \& Taylor L. T. (2004), Sequential Fractionation of Grape Seeds into Oils, Polyphenols, and Procyanidins via a Single System Employing CO2-Based Fluids, Journal of Agricultural and Food Chemistry, 52(9), pp. 2440-2444. doi:10.1021/jf030510n

15. Saykova I., Tylkowski B., Popovici C., \& Peev G. (2018), Extraction of phenolic and flavonoid compounds from solid wastes of grape seed oil production by cold pressing, Journal of Chemical Technology and Metallurgy, 53(2), pp. 177-190.

16. Singleton V. L., Orthofer R., \& Lamuela-Raventós R. M. (1999), [14] Analysis of total phenols and other oxidation substrates and antioxidants by means of folin-ciocalteu reagent, In: Methods in Enzymology (pp. 152-178). doi:10.1016/S0076-6879(99)99017-1

17. Ziyatdinova G. K., Nizamova A. M., \& Budnikov G. K. (2010), Galvanostatic coulometry in the analysis of natural polyphenols and its use in pharmacy, Journal of Analytical Chemistry, 65(11), pp. 1176-1180. doi:10.1134/S1061934810110146

18. Mazur L., Gubsky S., Dorohovych A., \& Labazov M. (2018), Antioxidant properties of candy caramel with plant extracts, Ukrainian Food Journal, 7(1), pp. 2313-5891. doi:10.24263/2304974X-2018-7-1-3

19. Gubskyi S., Nikitin S., Evlash V., \& Nemirich O. (2015). Iodine content determination in dried talli of laminaria by galvanostatic coulometry, Ukrainian Food Journal, 4(2), pp. 320-327.

20. Bezerra M. A., Santelli R. E., Oliveira E. P., Villar L. S., \& Escaleira L. A. (2008), Response surface methodology (RSM) as a tool for optimization in analytical chemistry, Talanta, 76(5), pp. 965-977. doi:10.1016/j.talanta.2008.05.019

21. Box G. E. P., \& Behnken D. W. (1960), Some New Three Level Designs for the Study of Quantitative Variables, Technometrics, 2(4), pp. 455-475. doi:10.1080/00401706.1960.10489912

22. Krishnaswamy K., Orsat V., Gariépy Y., \& Thangavel K. (2013), Optimization of MicrowaveAssisted Extraction of Phenolic Antioxidants from Grape Seeds (Vitis vinifera), Food and Bioprocess Technology, 6(2), pp. 441-455. doi:10.1007/s11947-012-0800-2

23. Apak R., Gorinstein S., Böhm V., Schaich K. M., Özyürek M., \& Güçlü K. (2013), Methods of measurement and evaluation of natural antioxidant capacity/activity (IUPAC Technical Report), Pure and Applied Chemistry: International Union of Pure and Applied Chemistry Journal, 85(5), pp. 957-998. doi:10.1351/PAC-REP-12-07-15

24. Prior R. L., Wu X., \& Schaich K. (2005), Standardized methods for the determination of antioxidant capacity and phenolics in foods and dietary supplements, Journal of Agricultural and Food Chemistry, 53(10), pp. 4290-4302. doi:10.1021/jf0502698

25. Budnikov G. K., \& Ziyatdinova G. K. (2005), Antioxidants as analytes in analytical chemistry, Journal of Analytical Chemistry. doi:10.1007/s10809-005-0146-2 